\title{
ON THE CORRECTNESS OF THE PROBLEM OF INVERTING THE FINITE HILBERT TRANSFORM IN CERTAIN AEROELASTIC MODELS
}

\author{
W.R. MADYCH
}

\begin{abstract}
We indicate methods of ensuring that the problem in the title is correctly posed in the $L^{p}$ sense whenever the derivative of the circulation function satisfies certain mild conditions.
\end{abstract}

1. Introduction. In the theory of aeroelastic control systems it is required to solve

$$
f(x)=\int_{-1}^{1} \frac{\gamma(y)}{y-x} d y
$$

for $\gamma(y),-1<y<1$, in terms of $f(x),-1<x<1$. The function $f$ is assumed to be of the form

$$
f(x)=w(x)+g(x)
$$

where

$$
g(x)=\int_{0}^{\infty} \frac{G(s)}{1-x+s} d s
$$

Here $w$ and $G$ are constant multiples of the so-called downwash function and the derivative of the circulation function respectively.

Formula (1) is often referred to as the finite Hilbert transform of $\gamma$, see [3]. For more detail concerning this model of aeroelasticity see [1] and the references cited there. In particular, $f=f^{t}$ and $G=\dot{\Gamma}_{t}$ in the notation of $[\mathbf{1}]$.

In order to guarantee the correctness of the problem of solving (1) via the methods in [3], it is necessary to assume that $f$ is in some $L^{p}(-1,1)$

Department of Mathematics, University of Connecticut, Storrs, CT 06268.

Partially supported by a grant from the Air Force Office of Scientific Research, AFOSR-86-0145. 
class. (If $J$ is an interval and $p$ is a positive number then $L^{p}(J)$ is the class of those Lebesgue measurable functions for which $\int_{J}|G(x)|^{p} d x$ is finite. When $p=\infty, L^{\infty}(J)$ is the class of essentially bounded functions on $J$.) In view of this and the fact that $w$ can usually be taken to be in any class $L^{p}(-1,1)$, it is important to obtain a fairly general answer to the following question: What conditions on $G$ ensure that $g$ is in $L^{p}(-1,1)$ ?

It is the purpose of this note to point out certain natural methods for obtaining such conditions. Propositions 2 and 3 below contain several typical results.

2. Discussion. Observe that the integral defining $g(x)$ is a smooth function of $x$ for $x<1$ whenever $G$ is locally integrable and satisfies a mild condition at infinity. In particular, it is clear that $g(x)$ is infinitely differentiable for $x<1$ if

$$
\int_{0}^{\infty} \frac{|G(s)|}{1+s} d s<\infty
$$

In fact, if $0<x<1$, we may write

$$
|g(x)| \leq(1-x)^{-1} \int_{0}^{\infty} \frac{|G(s)|}{1+s} d s
$$

and conclude that the only questionable behavior of $g$ occurs in arbitrarily small neighborhoods of $x=1$ whenever $G$ satisfies (4).

It should be noted that condition (4) is quite mild and general. For example, if $G=G_{1}+G_{2}$ where $G_{1}$ is in $L^{1}(0, \infty)$ and $G_{2}$ is in $L^{p}(0, \infty)$ for some $p, p<\infty$, then $G$ satisfies (4).

To understand how $G$ influences the behavior of $g$ in neighborhoods of $x=1$, express the integral defining $g$ as a sum, $\int_{0}^{\varepsilon}+\int_{\varepsilon}^{\infty}$, where $\varepsilon$ is any positive number $\leq 1$. Since

$$
\left|\int_{\varepsilon}^{\infty} \frac{G(s)}{1-x+s} d s\right| \leq \frac{2}{\varepsilon} \int_{0}^{\infty} \frac{|G(s)|}{1+s} d s,
$$

it should be clear that the behavior of $g$ at $x=1$ is determined by the behavior of $G$ at the origin. Indeed, if $s^{-\alpha} G(s)$ is in $L^{p}(0, \varepsilon)$ for some 
value of $p, 1 \leq p \leq \infty$, then by virtue of Hölder's inequality we may write

$$
\left|\int_{0}^{\varepsilon} \frac{G(s)}{1-x+s} d s\right| \leq I(x, p, \alpha)\left[\int_{0}^{\varepsilon}\left(s^{-\alpha} G(s)\right)^{p} d s\right]^{1 / p},
$$

where

$$
I(x, p, \alpha)=\left[\int_{0}^{\varepsilon}\left(\frac{s^{\alpha}}{1-x+s}\right)^{p /(p-1)} d s\right]^{1-1 / p}
$$

Now, using a change of variable, $I$ may be expressed as

$$
I(x, p, \alpha)=(1-x)^{\alpha-1 / p}\left[\int_{0}^{\varepsilon /(1-x)}\left(\frac{s^{\alpha}}{1+s}\right)^{p /(p-1)} d s\right]^{1-1 / p}
$$

from which we may easily estimate its size.

We summarize these observations as

Proposition 1. Suppose $g$ is related to $G$ via (3), $G$ satisfies condition (4), and $s^{-\alpha} G(s)$ is in $L^{p}(0, \varepsilon)$ for some positive $\varepsilon$, some $\alpha, \alpha \geq 0$, and some $p, 1 \leq p \leq \infty$. Then, for $-1<x<1$,

$$
|g(x)| \leq \begin{cases}C(1-x)^{\alpha-1 / p} & \text { if } \alpha<1 / p \\ C(1+\log (1-x)) & \text { if } \alpha=1 / p \\ C & \text { if } \alpha>1 / p\end{cases}
$$

where $C$ is independent of $x$.

A result concerning the $L^{p}$ class of $g$ follows as an immediate corollary.

Proposition 2. Suppose $G$ and g satisfy the hypothesis of Proposition 1 . If $\alpha \geq 1 / p$ then $g$ is in $L^{q}(-1,1)$ for all positive $q$. If $\alpha<1 / p$ then $g$ is in $L^{q}(-1,1)$ for all positive $q$ which satisfy $q<p /(1-\alpha p)$.

By using a slightly more delicate argument, the inequality $q<$ $p /(1-\alpha p)$ in the second half of the above proposition can be tightened 
to $q \leq p /(1-\alpha p)$ in the case $1<p<\infty$. To see this, use the fact that if $\alpha \geq 0$ and $x<1$ then

$$
\left(\frac{s}{1-x+s}\right)^{\alpha} \leq 1
$$

to observe that

$$
\left|\int_{0}^{\varepsilon} \frac{G(s)}{1-x+s} d s\right| \leq I_{\alpha} G_{\varepsilon}^{\alpha}(x-1)
$$

where

$$
I_{0} \phi(y)=\int_{-\infty}^{\infty} \frac{\phi(s)}{s-y} d s
$$

is the classical Hilbert transform of $\phi$ and, when $\alpha>0$,

$$
I_{\alpha} \phi(y)=\int_{-\infty}^{\infty} \frac{\phi(s)}{|s-y|^{1-\alpha}} d s
$$

is the fractional integral or Riesz potential of $\phi$. Here

$$
G_{\varepsilon}^{\alpha}(s)= \begin{cases}\left|s^{-\alpha} G(s)\right| & \text { if } 0<s<\varepsilon \\ 0 & \text { if } s<0 \text { or } s>\varepsilon\end{cases}
$$

The mapping properties of the transformation $\phi \rightarrow I_{\alpha} \phi$ are well known and, in view of (8), can be used to make conclusions concerning the behavior of $g$. For instance, if $1<p<\infty, 0 \leq \alpha<1 / p$, and $\phi$ is in $L^{p}(-\infty, \infty)$ then $I_{\alpha} \phi$ is in $L^{q}(-\infty, \infty)$ where $q=p /(1-\alpha p)$; see [2]. This, together with (5), (8), and Proposition 1, allows us to conclude

Proposition 3. Suppose $G$ and $g$ satisfy the hypothesis of Proposition 1 with the restriction that $1<p<\infty$ and $0 \leq \alpha<1 / p$. Then $g$ is in $L^{q}(-1,1)$ for all positive $q$ which satisfy $q \leq p /(1-\alpha p)$.

Another method of estimating the left hand side of (8) involves writing $\int_{0}^{\varepsilon}$ as $\int_{0}^{(1-x)}+\int_{(1-x)}^{\varepsilon}$ when $1-x<\varepsilon$, using the change of variables $y=1-x$, and applying variants of Hardy's inequality, see [2, 
p. 245] to each of the resulting integrals. This leads to generalizations of Proposition 1 for certain values of the parameters $\alpha$ and $p$.

For the sake of completeness we mention that, using similar methods involving fractional integrals, it is possible to obtain results concerning the behavior of $g$ in neighborhoods of 1 for other values of the parameters $\alpha$ and $p$. Since such estimates require the introduction of certain technical machinery and the conclusions do not involve $L^{p}$, we will not pursue the details here.

It may be worth noting that, in the notation of [1], $G(s)$ is equal to $\psi(t-s)$ for $t<s$; it is equal to another expression when $s<t$. Furthermore $\psi$ is assumed to be in $L^{1}(-\infty, 0)$. In this case our observations imply that if $t>0$ then $\psi$ does not affect the $L^{p}$ class of $f^{t}$. This should be compared with the conclusions in $[\mathbf{1}]$.

\section{REFERENCES}

1. J.A. Burns, E.M. Cliff, T.L. Herdman and J.Turi, On integral transforms appearing in the derivative of the equations of an aeroelastic system, in Nonlinear Analysis and Applications, V. Lakshmikantham, ed. Lecture Notes in Pure and Applied Mathematics, Volume 109, Marcel Dekker, Inc., New York 1987, 89-98.

2. G.H. Hardy, J.E. Littlewood and G. Polya, Inequalities, 2nd ed., Cambridge, 1952.

3. F.G. Tricomi, Integral equations, Interscience Publishers, Inc., New York, 1957.

Department of Mathematics, University of Connecticut, Storrs, CT 06268 
